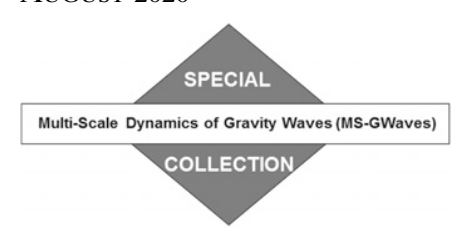

\title{
Transition from Geostrophic Flows to Inertia-Gravity Waves in the Spectrum of a Differentially Heated Rotating Annulus Experiment
}

\author{
COSTANZA RodDA AND UwE HARLANDER \\ Department of Aerodynamics and Fluid Mechanics, Brandenburg University of Technology Cottbus-Senftenberg, \\ Cottbus, Germany
}

(Manuscript received 2 February 2020, in final form 9 May 2020)

\begin{abstract}
Inertia-gravity waves (IGWs) play an essential role in the terrestrial atmospheric dynamics as they can lead to energy and momentum flux when propagating upward. An open question is to what extent IGWs contribute to the total energy and to the flattening of the energy spectrum observed at the mesoscale. In this work, we present an experimental investigation of the energy distribution between the large-scale balanced flow and the small-scale imbalanced flow. Weakly nonlinear IGWs emitted from baroclinic jets are observed in the differentially heated rotating annulus experiment. Similar to the atmospheric spectra, the experimental kinetic energy spectra reveal the typical subdivision into two distinct regimes with slopes $k^{-3}$ for the large scales and $k^{-5 / 3}$ for the small scales. By separating the spectra into the vortex and wave components, it emerges that at the large-scale end of the mesoscale the gravity waves observed in the experiment cause a flattening of the spectra and provide most of the energy. At smaller scales, our data analysis suggests a transition toward a turbulent regime with a forward energy cascade up to where dissipation by diffusive processes occurs.
\end{abstract}

\section{Introduction}

Atmospheric motions at the midlatitudes can be divided into synoptic-scale balanced motions, which develop from the baroclinic instability of the westerly flow, and mesoscale unbalanced motions, among which there are inertia-gravity waves (IGWs). It is now established that the balanced motions cannot exist without emitting IGWs in analogy to an elastic pendulum that cannot swing without fast axial oscillations (Vanneste 2013). This IGW generation process, observed in regions of the jet stream (O'Sullivan and Dunkerton 1995), is called "spontaneous emission" and it is considered a relevant source in the atmosphere together with other generation mechanisms like orography, convection, and boundary layer turbulence (Dörnbrack et al. 2018; Menchaca and Durran 2019; Polichtchouk and Scott 2020).

The synoptic- and mesoscale motions have distinct characteristics, and their time scales are usually well separated. The different dynamic regimes are reflected in the structure of the atmospheric kinetic energy spectra as shown by Nastrom and Gage (1985), who provided the first comprehensive spectra of the zonal

Corresponding author: Costanza Rodda,rodda@b-tu.de and meridional wind components and temperature measured near the tropopause level by commercial aircraft. The spectra exhibit two distinct power-law dependencies upon wavenumber in the form $\mathscr{P} \propto k^{p_{k}}$, where the typical measured values are $p_{k}=-3$ in the synoptic-scale range (for wavelengths between 500 and $3000 \mathrm{~km}$ ), and $p_{k}=-5 / 3$ in the mesoscale range (for wavelengths smaller than $500 \mathrm{~km}$ ). The steep slope corresponding to the -3 power law exhibited by the quasigeostrophic balanced flow, is consistent with Charney's theory of geostrophic turbulence (Charney 1971). Therefore, the hypothesis for this range of the spectrum is a downscale potential enstrophy cascade without any significant energy cascade. For wavelengths smaller than $500 \mathrm{~km}$, the spectral slope flattens and approaches $-5 / 3$; this suggests that at the mesoscale there is a different dynamical regime and some other phenomena are responsible for this part of the spectrum. Many investigations over the past years have focused on the mesoscale energy subrange trying to explain the phenomena involved and whether the energy is forward or inverse cascading (see, e.g., Lindborg 2007; Waite and Snyder 2009; Callies et al. 2014, and references therein). Observational evidence (Cho and Lindborg 2001) indicates a downward energy cascade at 
the small-scale end of the mesoscale (i.e., at the sub-100-km scales). Recently, Kafiabad et al. (2019) hypothesized that the power-law scaling of the shallow range could be explained with scattering of IGWs by geostrophic background flow. In their numerical results, however, they suggested that at smaller scales other more nonlinear phenomena with similar scaling dominate. These studies open the possibility of nonuniform dynamical regimes among the mesoscale range. Therefore, the energy source at the smaller scales remains a subject of debate. Although there is not a consensus on the nature of the mesoscale part of the spectra, the impact of spontaneously generated gravity waves on the midlatitude dynamics is well established, and it is, therefore, natural to include them among other possible sources (Dewan 1979; Callies et al. 2014; Žagar et al. 2017). Together with gravity waves, the other most invoked mechanisms are strongly stratified turbulence (Lindborg 2006) and quasigeostrophic turbulence (Tung and Orlando 2003).

A method that has been used to test the contribution of gravity waves to the energy consists of separating the kinetic energy spectrum into divergent and rotational parts (Bühler et al. 2014; Lindborg 2015). If the divergent part dominates and its ratio to the rotational component is larger than one, IGWs are the leading energy's contributors. By applying this separation method to subsets of the Measurement of Ozone and Water Vapor by Airbus In-Service (MOZAIC) dataset for the upper troposphere, Callies et al. (2014) found a dominance of the divergence spectrum at the mesoscale while Lindborg (2015) identified the rotational part as predominant and therefore concluded that stratified turbulence, rather than IGWs, is the prevailing mechanism. In the lower stratosphere, however, both investigations have shown that the mesoscale spectrum is clearly dominated by the divergence-IGW component. One possible reason behind these conflicting results is that although the energy spectra display global characteristics, the dynamics underlying the mesoscale energy spectrum may vary not only according to the height but also horizontally among different regions of the atmosphere (Bierdel et al. 2016).

In this paper, we propose a laboratory investigation of inertia-gravity waves emitted from baroclinic jets. One of the main advantages of using a laboratory experiment is that some gravity wave sources like orography and convection can be easily excluded. On the contrary, measured atmospheric data contain all sources of IGWs and it is often challenging to separate their contributions. The differentially heated rotating annulus experiment, a well-established experiment to study an analog dynamics of the atmosphere (Read et al. 2014), is used to generate baroclinic waves from which inertia-gravity waves are emitted (Hien et al. 2018; Rodda et al. 2018, 2019). The properties of the experimentally observed gravity waves are studied in detail together with the energy distribution among the balanced and imbalanced flow. The outcome of our investigation is a simplified picture of the atmospheric multiple-scale flow for which the relevant processes and spectral properties are more accessible than for atmospheric field data.

The paper is structured in the following way. In section 2, the experimental setup of the differentially heated rotating annulus and the measurement techniques are illustrated. Section 3 focuses on the gravity wave properties observed experimentally. The typical location of IGWs with respect to the jet front, their propagation velocity, horizontal scale, and frequencies are examined in detail. The energy distribution among the scales is investigated in section 4 . To evaluate the contribution of different phenomena, we separate the kinetic and total energy spectra into geostrophic and gravity wave components. At the end of the section, some considerations on the validity of the weakly nonlinear regime assumption used for the flow separation are discussed. A summary of the main findings and conclusions are given in section 5 .

\section{Experimental setup and data acquisition}

The experiment used in this work is an atmosphericlike differentially heated rotating annulus for which inertia-gravity waves emitted from the baroclinic jet have been observed both numerically (Borchert et al. 2014) and experimentally (Rodda et al. 2019). A plexiglass tank subdivided into three concentric cylinders, with inner radius $a=35 \mathrm{~cm}$ and outer radius $b=70 \mathrm{~cm}$, is mounted on a turntable and filled with deionized water. The water in the inner cylinder is kept to a constant low temperature by a system of pumps connected to a thermostat. Similarly, the water in the outer cylinder is kept to a constant higher temperature. The middle gap is our investigation region, and it is filled with water to a total depth of $H=6 \mathrm{~cm}$. The water in the gap, initially at room temperature, experiences a radial temperature difference imposed by the two insulated walls. Consequently, convective rolls covering the entire fluid depth develop. For high enough rotation rates, the flow becomes baroclinically unstable and forms baroclinic waves. A more detailed description of the experiment and the flow regimes can be found in Rodda et al. (2019).

The set of experiments presented in this paper is run with the lateral temperature difference set to $\Delta T=4.8 \mathrm{~K}$ and the rotation rate varying from $\Omega=0.5$ to $1 \mathrm{rpm}$. The measurement technique used to investigate 
the flow velocity in the midgap is the nonintrusive twodimensional particle image velocimetry (PIV). The seeding particles are hollow glass spheres with mean particle size $10 \mu \mathrm{m}$ and density $1.1 \mathrm{~g} \mathrm{~cm}^{-3}$. A diodepumped steady laser (model LINOS nano; max power: $100 \mathrm{~mW}$; wavelength: $532 \mathrm{~nm}$ ) is fixed to the tank and corotates with it. The laser beam goes through a cylindrical-lens optical system that spreads it into a light plane of thickness $\approx 1 \mathrm{~mm}$, which is small enough to be approximated to a two-dimensional plane. The area illuminated by the laser (covering approximately $1 / 6$ of the annulus), is recorded by a corotating camera (GoPro hero 4 black with video resolution set to $1080 \mathrm{p}$ and $48 \mathrm{fps})$. The camera is updated with a lens (The Imaging Source, model TCL 1216) that allows for control on the focus and the aperture. The recorded videos have a total duration between 45 and $50 \mathrm{~min}$ for each experimental run.

The frames recorded by the camera are successively processed with sophisticated image-processing techniques that calculate the displacement of the particle pattern between two images and output the fluid velocity horizontal components. For this data processing, we use the free MATLAB toolbox UVmat, developed at Laboratoire des Écoulements Géophysiques et Industriels (LEGI) in Grenoble (downloadable at http:// servforge.legi.grenoble-inp.fr/projects/soft-uvmat). The complete documentation about the use of the software can be found in Sommeria (2003). Finally, the two horizontal velocity components $u$ and $v$ are organized on a regular Cartesian grid with a spatial resolution of $0.2 \mathrm{~cm}$ on both $x$ and $y$ axes. Furthermore, a time average is applied to the data resulting in the final temporal resolution of $0.5 \mathrm{~s}$. The smoothing in time is done to reduce the noise level at the high frequencies, which correspond to the very small scales. The smoothing does not affect the gravity waves that are well resolved in space and time.

The vertical temperature profile is measured locally by temperature sensors [thermocouple sheathed sensors consisting of two wires $\left(\mathrm{NiCr}-\mathrm{Ni}\right.$ ) with resolution $\left.0.1^{\circ} \mathrm{C}\right]$ positioned at midgap at different fluid depths. The vertical temperature difference $\Delta_{z} T$ is used to calculate the buoyancy frequency $N=\left(g \alpha \Delta_{z} T / H\right)^{1 / 2}$, where $g$ is gravity, $\alpha$ the thermal expansion coefficient, and $H$ the total fluid depth. Our previous study (Rodda et al. 2019) on the horizontal distribution of $N$ in experiments with the same setup has shown the relevance of such distribution for the emission and propagation of gravity waves. Keeping in mind that the baroclinic wave slowly propagates with respect to the corotating temperature sensors, the spatial variation of $N$ is well captured by the measured time dependency, which gives
$0.15<N<0.30 \mathrm{rads}^{-1}$. In the following discussion, however, the mean value $N=0.22 \mathrm{rad} \mathrm{s}^{-1}$ will be used to calculate the upper limit for the gravity wave frequency.

\section{Inertia-gravity waves}

Inertia-gravity waves, due to their small spatial and short temporal scales, are challenging to observe directly in the velocity field, which is dominated by the large-scale flow. However, since in good approximation the balanced flow can be considered divergence-free, the imbalanced part of the flow can be identified as wave pattern in the horizontal divergence. For this reason, many studies employ the horizontal divergence as a dynamical indicator of IGWs (O'Sullivan and Dunkerton 1995; Plougonven et al. 2003; Wu and Zhang 2004; Dörnbrack et al. 2012; Khaykin et al. 2015).

A snapshot of the horizontal divergence calculated from the horizontal velocity components (measured at a fluid height $z=5 \mathrm{~cm}$ for an experimental run with $\Omega=$ $0.5 \mathrm{rpm}$ ) is plotted in Fig. 1 in the inset on the left. The plot is superimposed to the surface temperature map measured with an IR camera. Such combined plot displays the small-scale features position at the exit region of the large-scale baroclinic wave (see sketch representing the jet exit and entrance regions in Fig. 1). The largest amplitude in the horizontal divergence signal is concentrated along the jet front and exhibits wavelike structures with the wave crests roughly perpendicular to the flow. The waves appear irregularly, propagate within the jet stream, are mostly advected by it, and then dissipate; we refer to this irregular behavior as intermittency. The small waves present similar characteristics also for the other experimental runs at different rotation rates varying in the range $0.5<\Omega<1 \mathrm{rpm}$. In all experiments, the regimes explored are characterized by baroclinic waves with a dominant spatial structure and azimuthal wavenumber $m=5,6$, or 7 depending on the rotation rate. However, in some cases, we have steady wave regimes while in others, the waves show amplitude vacillations. The situation for full geostrophic turbulence is not included in the present study.

A rough estimate of the zonal and meridional horizontal wavelength from Fig. 1 gives $\lambda_{x} \approx 5 \mathrm{~cm}$ and $\lambda_{y} \approx$ $4.5 \mathrm{~cm}$, in the local Cartesian system of reference, where $y$ points in the radial direction. If we compare these wavelengths with the typical length scale of the baroclinic vortices, which is given by the Rossby deformation radius $L_{D}=N H / f \approx 12 \mathrm{~cm}$, it emerges that the characteristic length scales of IGWs are approximately 3 times smaller than that of the core region of the baroclinic vortices. These findings are in agreement 


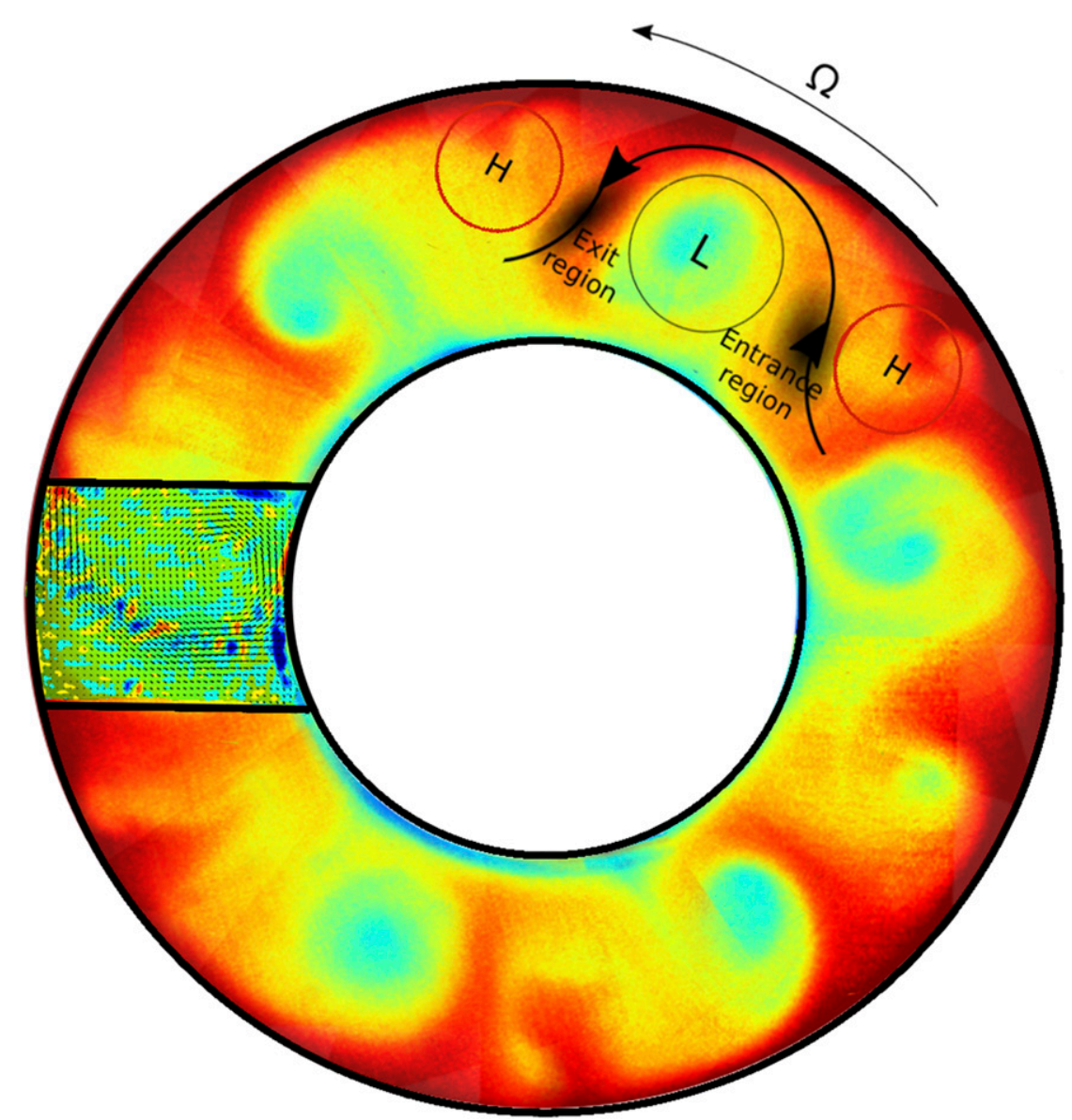

FIG. 1. Combined plot of the surface temperature reconstructed from measurements done with the infrared camera, and the velocity field (black arrows). The sketch illustrates the position of the entrance and exit regions of the jet. The baroclinic wave is in a regular regime with azimuthal wavenumber $m=6$. The colors in the small inset show the horizontal divergence measured by PIV. Small-scale wavelike features are recognizable in the horizontal divergence field; they appear along the baroclinic jet and propagate with it.

with Kafiabad and Bartello (2018), who showed that the spontaneous generation occurs at scale smaller but comparable to the most energetic scales of the balanced flow.

The small-scale waves propagating along with the jet qualitatively resemble the atmospheric inertia-gravity waves observed along jets and fronts. In particular, observations but also numerical models indicate the jet exit region and also, although less frequently, the entrance region as favored locations for large-amplitude IGWs (see, e.g., Plougonven and Zhang 2014, and references therein; Dörnbrack et al. 2018; von Storch et al. 2019).

Another important characteristic of the jet's exit region is the large deformation of the flow due to its deceleration. Plougonven and Snyder (2005) observed that regions with intense horizontal deformation and vertical shear can be crucial for the gravity wave propagation giving rise to a phenomenon called wave capture (Bühler and McIntyre 2005). When wave capture occurs, the horizontal wave vectors tend to align with the contraction axis of the flow, whereas the tilt of wave vectors tends to converge to a value given by the ratio of vertical shear and deformation (Wang et al. 2009). It can be noticed in Fig. 1 that the wave trains have, indeed, horizontal wave vectors that tend to orient along the contraction axis, which is aligned with the jet, and occur in regions where the deformation is the largest. This investigation reveals that the IGWs emitted from the baroclinic flow in our experiment likely undergo wave capture during their propagation through the flow (Rodda 2019).

\section{IGWs dispersion relation}

To further investigate the wave properties, we perform an analysis in Fourier space. The aim is to identify 


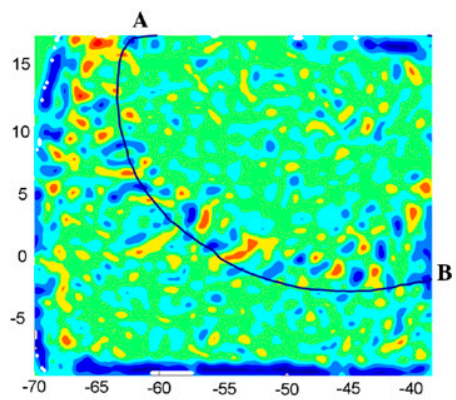

(a)

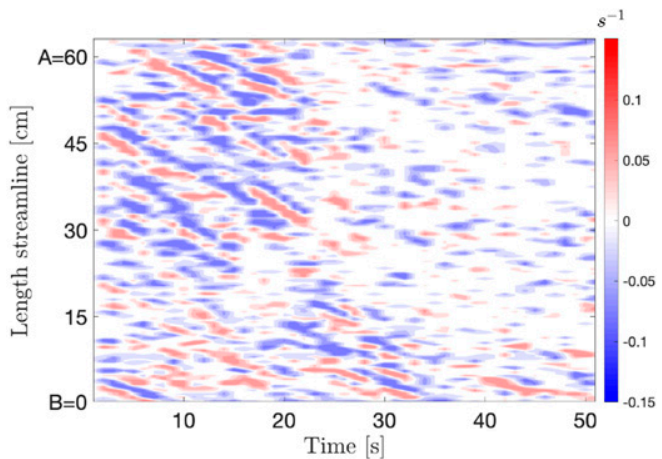

(c)

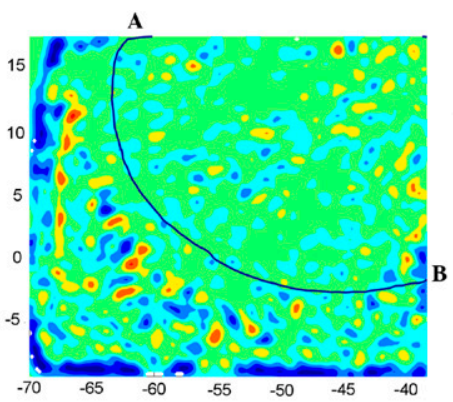

(b)

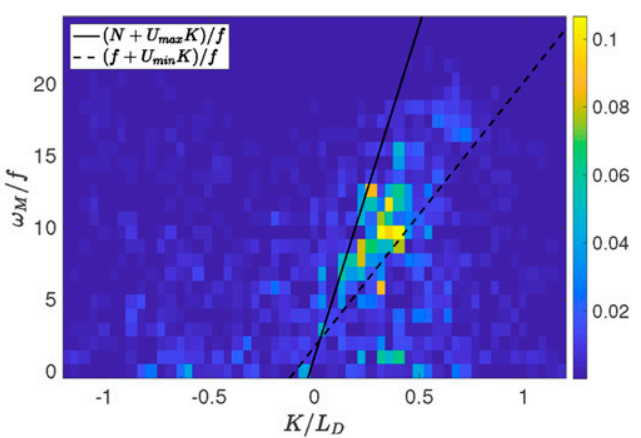

(d)

FIG. 2. Analysis of the frequencies and wavenumbers. (a),(b) The plot of the horizontal divergence and the streamline at $t_{0}=0$ and $t_{1}=40 \mathrm{~s}$. (c) Hovmöller plot of the horizontal divergence for $1 \leq t \leq 50 \mathrm{~s}$, and (d) $2 \mathrm{D}$ Fourier transform of (c). The measured frequencies and wavenumbers are normalized by the Coriolis frequency $f=$ $0.13 \mathrm{rad} \mathrm{s}^{-1}$ and the Rossby deformation radius $L_{D}=12 \mathrm{~cm}$. The black solid and dashed lines depict the upper and lower limits for IGWs, which are $f$ and $N=0.22 \mathrm{rad} \mathrm{s}^{-1}$ Doppler shifted using the maximum velocity $U_{\max }=$ $0.5 \mathrm{~cm} \mathrm{~s}^{-1}$ and minimum velocity $U_{\min }=0.2 \mathrm{~cm} \mathrm{~s}^{-1}$ measured along the jet and multiplied by $-15<K<15 \mathrm{~cm}^{-1}$, respectively.

the wave frequencies and see if they fulfil the IGWs dispersion relation

$$
\omega_{i}^{2}=\frac{N^{2} K_{H}^{2}+f^{2} n^{2}}{K_{H}^{2}+n^{2}},
$$

where $\omega_{i}$ is the intrinsic frequency, $N$ the buoyancy frequency, $f=2 \Omega$ the Coriolis frequency, $K_{H}=\sqrt{k^{2}+l^{2}}$ the horizontal wavenumber, and $n$ the vertical wavenumber. Frequencies and wavenumbers of the measured waves can be obtained by calculating the space-time data Fourier transform. The two-dimensional data for such Fourier analysis consist of the horizontal divergence sampled along the jet over time. This choice is the most convenient considering that, as we have previously shown, the small-scale waves are aligned with the jet and propagate within it. The data along the jet are calculated by interpolating the horizontal divergence along a streamline frozen in time, identifying periods where the jet front is passing over the chosen streamline. Constant values of the streamfunction $\Psi$, which is related to the horizontal velocity components as $u=$ $\partial_{y} \Psi$ and $v=-\partial_{x} \Psi$, define the streamlines. The chosen streamline (black line), identifying the baroclinic front at $t=t_{0}$, and the horizontal divergence data (color map) are plotted in Figs. 2a and 2b for $t_{0}$ and $t_{1}=t_{0}+40 \mathrm{~s}$, respectively.

The horizontal divergence is then interpolated along this curve for the time interval considered and successively displayed in a time-space Hovmöller plot (see Fig. 2c). The orientation of the divergence signal in Fig. $2 \mathrm{c}$ shows no alterations in the slope, meaning that the waves propagate with the same angle to the jet and with a constant phase speed. Moreover, the signal fades in time, showing that the strongest waves are embedded in the jet since when the baroclinic front moves farther downstream, the signal becomes weaker and is lost later.

From the data plotted in Fig. 2c, we can finally proceed to the Fourier space (Fig. 2d) and analyze the horizontal wavelengths and frequencies (Yarom and Sharon 2014). The wavenumber of the divergence along the streamline is on the $x$ axis, while the observed 
frequencies normalized by the Coriolis frequency $\omega_{M} / f$ are on the $y$ axis. The first feature that can be noticed is an asymmetry in the distribution of the peaks, showing a prominent signal on the right-hand side of the plot, i.e., for positive wavenumbers. This asymmetry is due to the Doppler shift. Indeed, the measured frequency is the result of the IGW intrinsic frequency $\left(\omega_{i}\right)$ summed to the Doppler term $\left(\mathbf{U}_{0} \cdot \mathbf{K}_{H}\right)$, which account for the propagation of the mean flow

$$
\omega_{M}=\omega_{i}+\mathbf{U}_{0} \cdot \mathbf{K}_{H} .
$$

According to (2), the asymmetry can be interpreted as the preferential direction of propagation in the jet flow direction. For waves propagating against the flow, one would instead observe the negative branch. It follows from the dispersion relation that the frequencies span the gravity wave range $f+\mathbf{U}_{0} \cdot \mathbf{K}_{H}<\omega_{M}<N+\mathbf{U}_{0} \cdot \mathbf{K}_{H}$. The solid and dashed black curves mark these upper and lower limits in Fig. 2d. The horizontal wavenumber is assumed to vary in the interval -15 to $15 \mathrm{~cm}^{-1}$, which is the range spanned by the measured gravity waves. The Doppler shift is estimated from the two horizontal velocity components $u$ and $v$ interpolated along the chosen streamline. The magnitude is then calculated as $U=$ $\left(u^{2}+v^{2}\right)^{1 / 2}$ and its minimum and maximum values $U_{\min }=0.2 \mathrm{~cm} \mathrm{~s}^{-1}$ and $U_{\max }=0.5 \mathrm{~cm} \mathrm{~s}^{-1}$ are added to $f=0.13 \mathrm{rad} \mathrm{s}^{-1}$ and $N=0.22 \mathrm{rads}^{-1}$, respectively. The two resulting curves delimit the region with the most significant amount of energy, indicating that the energy peaks are, indeed, related to gravity waves.

Although some IGW generation mechanisms relevant to the atmosphere can be excluded in the laboratory experiment, an exhaustive investigation of all the possible sources is challenging, as the full three-dimensional velocity field is not available. The horizontal divergence field is an appropriate choice for identifying inertiagravity waves and study their characteristics, but it has the limitation that the field could still incorporate a nonnegligible part of the balanced flow. A more precise separation of the balanced and unbalanced part of the flow becomes, therefore, of fundamental importance when studying spontaneous imbalance processes. Sophisticated methods involving a modal decomposition of the full field to separate balanced and unbalanced fields have been applied, for example, by Hien et al. (2018) for a linear system and by Kafiabad and Bartello (2017) and Chouksey et al. (2018) for nonlinear systems. Unfortunately, these diagnostic tools require the full three-dimensional velocity field and are, therefore, often impossible to use for laboratory data or observations that generally do not give the full three-dimensional information. For this reason, with our experimental data, we can only investigate whether some sources can be excluded and speculate on which sources are more likely to be responsible for the emission of IGWs.

Among the possible sources of gravity waves in the rotating annulus, there are shear instabilities. To investigate such instability, the Richardson number $\mathrm{Ri}=$ $\left(N / \partial_{z} U\right)^{2}$ is estimated from the buoyancy frequency $N$, which is calculated by the vertical temperature profile measured with sensors, and the velocity shear $\left(\partial_{z} U\right)$ calculated by PIV measurements at different fluid heights. Since $N$ is derived from temperature sensors positioned along points on a vertical line in the center of the gap, the temporal mean azimuthal velocity $(U)$ is calculated at the same locations. Then, the maximum and minimum values of $N$ are taken to find the corresponding values for the Richardson number are $9.7<$ $\mathrm{Ri}<39$, a range that is much higher than $\mathrm{Ri}_{\text {critical }}=0.25$. Although the measurements for estimating these parameters can only be done locally, the horizontal distribution of the Richardson number has been calculated using corresponding numerical simulations [see Rodda et al. (2019) for details] and the range of values is in excellent agreement with the one found for the laboratory experiment. Therefore, shear instabilities can be ruled out as wave sources for the investigated regions of the baroclinic wave.

In the experiment, another possible source of gravity waves comes from the boundary instabilities at the inner cylindrical wall (Jacoby et al. 2011; Randriamampianina and del Arco 2015; von Larcher et al. 2018; Hien et al. 2018). Although waves have been reported to be generated from the inner wall and then propagate toward the middle part of the gap, we speculate that such a mechanism is not of primary relevance for our experiment. Our speculation relies on the wave characteristics observed in our case differing from the ones observed by von Larcher et al. (2018) in a small tank. More specifically, in von Larcher et al. (2018) gravity waves propagate retrograde with respect to the baroclinic flow while the opposite is true in our case. Furthermore, the waves in our experiment are continuously generated along the baroclinic jet and then propagate along with it rather than entering the jet from the inner wall. Finally, the numerical simulations by Hien et al. (2018) for a setup of the differentially heated rotating annulus analogous to our experiment (even though their IGW fields differ from ours) evinced that most of the gravity waves in the core region of the baroclinic wave are spontaneously generated.

From the arguments exposed, we suppose that a substantial part of the IGWs is likely to be spontaneously emitted from the baroclinic jets. This thesis is also supported by Rodda et al. (2019), where we showed a 
correlation between the size of the Rossby number and the region of IGW emission.

\section{Energy spectra}

After discussing the characteristics of the gravity waves emitted from the baroclinic jet, we now investigate the energy distribution among the scales. The idea is to analyze the experimental data with the same methods used for atmospheric data and see if the overall spectra show the same global characteristics. The reference atmospheric spectra considered here are from Nastrom et al. (1984), who calculated wind and temperature spectra from measurements taken from over 6900 commercial air flights at the midlatitudes. These spectra have been extensively cited in the literature and referred to as a reference for many successive studies aiming to explain the underlying physical phenomena. Although in our experiment the velocities are measured in two dimensions, and therefore it would be possible to calculate 2D space spectra, the field of view of the PIV is rather small (see Fig. 1). For this reason, only the dynamics at the small scales can be investigated by computing the spatial spectra. Calculating the frequency spectra from the measured velocity time series and then convert them into wavenumber spectra, on the other hand, gives access to a broader scale range that also covers the dynamics at the larger scales. In this way, the experimental spectra can be compared with the atmospheric spectra by Nastrom et al. (1984). To convert frequencies into wavenumbers, the Taylor hypothesis has to be valid for the experimental data (Taylor 1938). Note that also ship- and flight-track data recently discussed by Callies et al. (2014) have been sampled in time and are converted later to give spatial information. As we discussed in the previous section, one of the main characteristics of the observed IGWs is their propagation along the jet, mostly advected by it. This peculiar behavior allows us to apply Taylor's hypothesis if we consider the jet as a mean velocity field of constant velocity $U_{\max }$. In this way, the transformation $E(f)=E(k)(2 \pi) / U_{\max }$ applies, where $E(f)$ is the frequency power spectrum and $E(k)$ the one-dimensional wavenumber spectrum and $f \approx U_{\max } k / 2 \pi$ (Kumar and Verma 2018).

The dataset is prepared by removing the data close to the boundary in the 2D plane (see Fig. 2a), which are affected by larger uncertainties. This operation leaves us with a dataset consisting of 8132 time series. The power spectral density for each horizontal velocity component $\left(\hat{C}_{u}\right.$ and $\left.\hat{C}_{v}\right)$ is calculated for every point in the $2 \mathrm{D}$ plane. Subsequently, a mean spectrum is derived from all the spectra so that local effects are smoothed out.

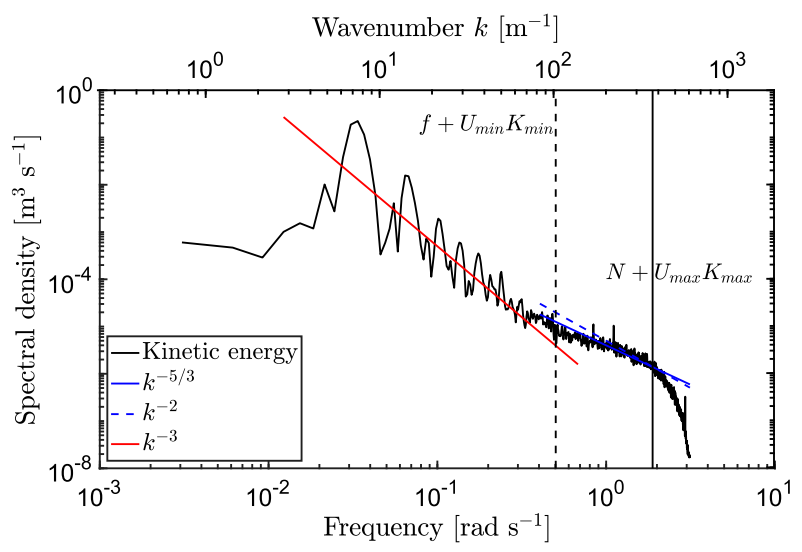

FIG. 3. Mean kinetic energy spectrum. The red line displays the $k^{-3}$ power law, the blue line is $k^{-5 / 3}$, and the blue dashed line is $k^{-2}$. The vertical dashed and solid lines show the minimum and the maximum Doppler shifted frequencies for inertia-gravity waves, respectively. The minimum dashed line is calculated via $\omega_{\min }=f+$ $U_{\min } K_{\min }$, where $f=0.13 \mathrm{rad} \mathrm{s}^{-1}$ and the Doppler shift $U_{\min } K_{\min }=$ $0.38 \mathrm{rad} \mathrm{s}^{-1}$. The maximum solid line is calculated via $\omega_{\max }=N+$ $U_{\max } K_{\max }$, where $N=0.22 \mathrm{rad} \mathrm{s}^{-1}$ and the Doppler shift $U_{\max } K_{\max }=$ $1.51 \mathrm{rad} \mathrm{s}^{-1}$. Note that these lines are analogous to the curves plotted in Fig. $2 \mathrm{~d}$ for constant $K_{\min }$ and $K_{\max }$ measured.

Similar averaging procedures have also been applied by Nastrom et al. (1984) and Callies et al. (2014).

The kinetic energy spectra have been calculated for different experimental runs where the lateral temperature difference is kept constant to $\Delta T=4.8 \mathrm{~K}$, but the rotation rate varies in the range $\Omega=0.5$ to $1 \mathrm{rpm}$. The spectra for all the cases (not reported here for brevity) look qualitatively similar to the one generically chosen $(\Omega=0.5 \mathrm{rpm})$ in Fig. 3. A common problem that emerged from the analysis is that longer time series would be needed to resolve the low-frequency part of the spectra fully. The authors believe that the high peak oscillations at the low frequency in Fig. 3 are an artifact arising from these scales not being fully resolved. Nevertheless, in all the measurements, there is a clear transition from a steeper to a shallower slope. In the following, we will discuss further results for the selected case that shows a steady baroclinic wave $m=6$. The analysis has been done for all the other dataset as well and, since the results are comparable, shall not be reported in detail here.

As previously discussed, the kinetic energy spectra calculated for our experiment (Fig. 3) reveal a striking resemblance with typical atmospheric spectra (Nastrom et al. 1984). Although the large scales are not entirely resolved (as even longer time series would be needed), an evident change of slope is visible at frequencies around $\omega=0.4 \mathrm{rad} \mathrm{s}^{-1}$. For the flatter part, two slopes are depicted: $-5 / 3$ and -2 . The latter is the slope based upon the theory by Garrett and Munk (1979) for internal gravity waves in the ocean. We mention the 
Garret-Munk model because the internal gravity wave model for the atmosphere that we use is, in fact, an extension of the well established oceanic model. VanZandt (1982) showed that for the atmospheric spectra, the slope should be changed from -2 to $-5 / 3$. Although the two slopes are not very far from one another, as it can be seen in Fig. 3, the $-5 / 3$ fits our data almost perfectly. The better fit of the $-5 / 3$ slope suggests that, concerning the energy spectrum in the investigated regime, the experiment has more features in common with the atmosphere than with the ocean. As mentioned earlier, due to the limited extension of the observation area the spatial spectra can only be directly calculated for this small-scale range. The two-dimensional $k_{x}$ and $k_{y}$ spectra averaged over time are calculated and then integrated over the horizontal wavenumber $K_{H}=\sqrt{\left(k_{x}^{2}+k_{y}^{2}\right)}$. The resulting spectra (not reported here for brevity) show a $K_{H}^{-5 / 3}$ slope. This cross-check confirms the validity of the Taylor's hypothesis for the small-scale features. The sharp steepening of the spectrum for the highest frequencies is an artifact related to the time smoothing used in the PIV post processing. Since the artificial steepening starts at scales outside the gravity waves regime, the results are not affected by the drop. The vertical lines in Fig. 3 mark the range of frequencies of IGWs. The lower limit, indicated by the dashed line, is calculated as $\omega_{\min }=f+$ $U_{\min } K_{\min }$, where $f=0.13 \mathrm{rad} \mathrm{s}^{-1}$ and the Doppler shift $U_{\min } K_{\min }=0.38 \mathrm{rad} \mathrm{s}^{-1}$. The upper limit, indicated by the solid line, is calculated $\omega_{\max }=N+U_{\max } K_{\max }$, where $N=0.22 \mathrm{rad} \mathrm{s}^{-1}$ and the Doppler shift $U_{\max } K_{\max }=$ $1.51 \mathrm{rad} \mathrm{s}^{-1}$. Note that these lines are analogous to the ones plotted in Fig. 2d, but this time the wavenumber minimum and maximum values $\left(K_{\min }\right.$ and $\left.K_{\max }\right)$ evaluated from Fig. $2 d$ are considered. It can easily be seen that the flatter subrange of the spectrum lies within the IGWs frequency range. This already hints that gravity waves might substantially contribute to the energy at these scales.

\section{a. Spectra decomposition}

To understand better the energy distribution over the multiple scales present in the laboratory experiment, we use the spectral decomposition method developed by Bühler et al. (2014). This method separates the kinetic energy spectra into the divergent and rotational part of the flow. A similar approach has been proposed by Lindborg (2015), and both methods have been applied to atmospheric data collected by aircraft (MOZAIC dataset). These two decomposition methods have been tested by Bierdel et al. (2016) on identical datasets, and the results are almost indistinguishable. Based on this equivalence, we opted for the method developed by Bühler et al. (2014) because it offers a further decomposition in wave and vortex components of the flow, based on the assumption that gravity waves are in a nearly linear regime.

The wave vortex decomposition method by Bühler et al. (2014), consisting of a two-step decomposition, is briefly described first and then applied to our experimental data. Such decomposition applies to the along-track $u$ and cross-track $v$ velocities measured by the aircraft. In our experiment, $u$ and $v$ are defined as the azimuthal and radial velocity components. Since the flow moves prograde to the PIV measuring system (which is corotating with the tank), the components are equivalent to the along-track and cross-track velocities measured by a plane moving along the azimuthal $u$ direction. The first step is a Helmholtz decomposition performed over the horizontal velocity power spectra $\left(\hat{C}^{u}\right.$ and $\left.\hat{C}^{v}\right)$ to separate the rotational $K^{\psi}(k)$ and the divergent component $K^{\phi}(k)$ in the following form:

$$
\begin{aligned}
& K^{\psi}(k)=\frac{\hat{C}^{v}(k)}{2}+\frac{1}{2 k} \int_{k}^{\infty}\left[\hat{C}^{v}(\bar{k})-\hat{C}^{u}(\bar{k})\right] d \bar{k}, \\
& K^{\phi}(k)=\frac{\hat{C}^{u}(k)}{2}-\frac{1}{2 k} \int_{k}^{\infty}\left[\hat{C}^{v}(\bar{k})-\hat{C}^{u}(\bar{k})\right] d \bar{k} .
\end{aligned}
$$

Since we have a discrete dataset, the integrals are calculated numerically via the trapezoidal method implemented in the MATLAB function trapz, where the integration area is broken down into trapezoids spaced by the distance between each sampled point.

Figure 4a presents the Helmholtz decomposition for the energy spectra, obtained from (3a) and (3b). The kinetic energy is plotted in black, the rotational component $K^{\psi}$ in (3a) in red, and the divergent component $K^{\phi}$ in (3b) in blue. The blue and the red curves intersect at a frequency equal to $0.4 \mathrm{rad} \mathrm{s}^{-1}$, which corresponds to the frequency where the slope flattens in Fig. 3c. Another feature visible in Fig. 4a and later in Fig. 4b is that the divergent energy spectrum, attributed to the subsequently calculated wave energy spectrum, is truncated at small frequencies. This truncation occurs because the decomposition method is based on the assumption that the velocities are isotropic. As Bühler et al. (2017) recently evinced, if the data are anisotropic, the divergent energy spectrum becomes negative at small wavenumbers and this results in a data gap, similar to what is observed in the plots presented here. To resolve this problem, Bühler et al. (2017) developed a method that, under certain conditions, can be applied to anisotropic data. This anisotropic method involves the further calculation of the cross-spectrum $\hat{C}^{u v}$, and it can be applied under the assumptions that the 


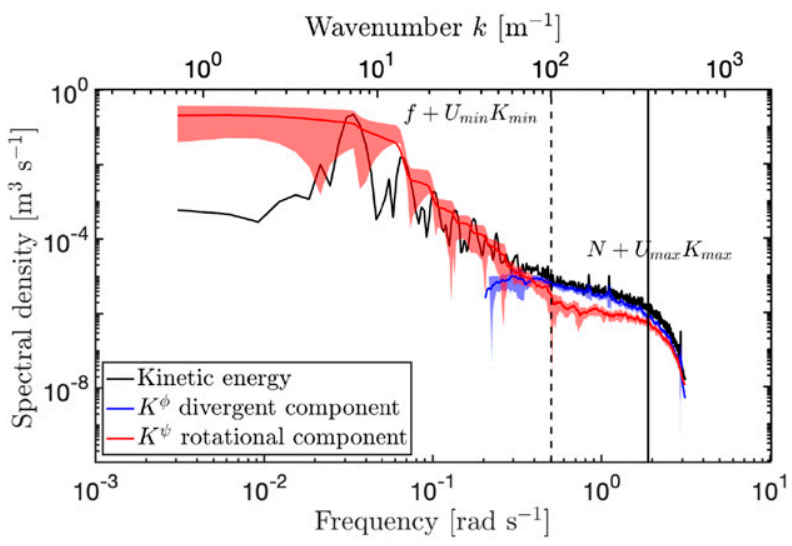

(a)

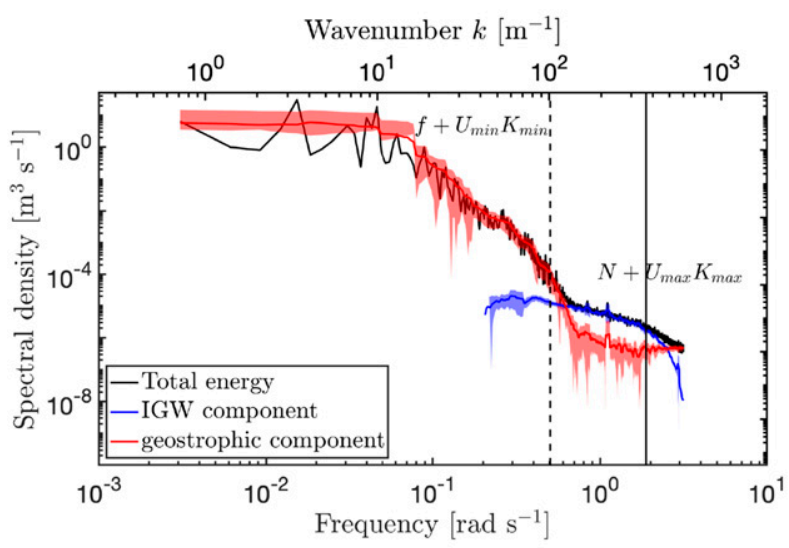

(b)

FIG. 4. Energy spectra decomposition. (a) Kelvin-Helmholtz decomposition. The black curve shows the spectra of the kinetic energy, the red curve is the rotational component [see (3a)], and the blue curve is the divergent component [see (3b)]. (b) Gravity waves residual geostrophic component decomposition. The black curve shows the total energy spectrum calculated by adding the potential energy in (4) to the kinetic energy spectrum, the blue curve is the wave component $E_{W}(k)$ calculated in (5), and the red curve is the residual geostrophic component calculated by subtracting $E_{W}(k)$ from the total energy. The dashed and solid lines are calculated as in Fig. 3. In both plots, the red and blue curves show the data smoothed with a moving average calculated over 20 data points, while the colored shadows represent $2 \sigma$ variance.

imaginary part $\Im\left(\hat{C}^{u v}\right)$ is negligible and the real part $\Re\left(\hat{C}^{u v}\right)$ is sign-definite. However, both conditions are not fulfilled for our dataset, and therefore the anisotropic method cannot be used. Even so, the isotropic separation method gives results that fully cover the flatter part of the spectrum (on which our analysis is mostly focused) and additionally reaches deep into the frequency part where the rotational component has a steep slope. Hence, the region where the rotational and the divergent part intersect is well resolved.

The rotational component (red line in Fig. 4a) is the one contributing the most to the energy for small frequencies, while the divergent component (blue line) becomes more important at frequencies in the gravity wave range. If we translate frequencies into wavenumbers (visible on the top $x$ axis in Figs. 4a,b), this plot clearly shows that the divergent component matters at small scales. This decomposition highlights a difference between the laboratory and the atmosphere as the divergent and rotational spectral component of the first are well separated in the $-5 / 3$ regions while they are in the same order of magnitude for the latter, as shown by Callies et al. (2014). The two components being comparable is the reason for Lindborg (2007) to argue that stratified turbulence is more likely responsible for the flattening of the atmospheric spectrum in the upper troposphere. In our case, on the other hand, the results point to gravity waves as the main energy source, which suggests a scenario more similar to what is observed in the lower stratosphere regions.

The second step of the Bühler et al. (2014) method consists in decomposing the total energy spectrum into the geostrophic and inertia-gravity wave components. With respect to the Helmholtz decomposition, this further step accounts for inertia-gravity waves made of both a divergence and a rotational component, and it is based upon the linear dynamics of inertia-gravity waves (we shall discuss the validity of this assumption for our data in the next section). For calculating the total energy spectrum, we need to consider the potential energy in addition to the already calculated kinetic energy. From the temperature measurements collected via temperature sensors placed in the middle of the tank, we could determine the corresponding potential energy spectrum, which reads

$$
\hat{C}^{b}(k)=|\hat{b}(k)|^{2} / N^{2},
$$

where $b$ is the local buoyancy and $N$ the buoyancy averaged over space and time.

By further assuming that the field is composed of plane waves, from which vertical homogeneity follows, we can use the dispersion relation of gravity waves in (1) to derive the equipartition relation for the spectra as

$$
\hat{C}^{b}(k)+2 K^{\psi}(k)=2 K^{\phi}(k)+\hat{C}_{W}^{w}(k) \approx E_{W}(k),
$$

where $\hat{C}_{W}^{w}(k)$ is the vertical kinetic energy. If we assume that $\hat{C}_{W}^{w}(k)$ is negligible, the hydrostatic internal wave energy spectrum reduces to $E_{W}(k) \approx 2 K^{\phi}(k)$. 
The total energy decomposition in Fig. $4 \mathrm{~b}$ further confirms that the small scales are dominated by nearly linear gravity waves, whose energy is comparable to the total energy spectrum in the $-5 / 3$ range. The comparison of Figs. 4a and 4b evinces similar energy parts for the divergence and the inertia-gravity wave component, also indicating that here the horizontal component is the most energetic part of the gravity waves.

In both decompositions the rotational (see red curve in Fig. 4a) and geostrophic (see red curve in Fig. 4b) component keep having a steep slope at the mesoscales, but do eventually flattens toward a $-5 / 3$ slope parallel to the divergent and gravity wave spectral component (see red curve in Figs. 4a and 4b, respectively). An analogous behavior has been reported by Callies et al. (2014) and the authors described this flattening as an artifact and attributed this to interpolation procedure and truncation errors. On the other hand, some numerical studies reported analogous features with the geostrophic spectrum approaching a $-5 / 3$ slope for very small scales and associated this behavior to a transition toward an isotropic turbulence regime (Bartello 2010; Kafiabad and Bartello 2016).

\section{b. Weakly nonlinear regime approximation}

We have seen in the previous section that the kinetic energy spectrum consists of a divergent and a rotational part $E_{\text {kin }}=K^{\phi}+K^{\psi}$, where $E_{\text {kin }}$ is the kinetic energy. The ratio between the two components, defined as

$$
R=\frac{K^{\phi}}{K^{\psi}}
$$

gives another insight about the dynamics at the mesoscale. Indeed, realistic frequency distributions of linear gravity waves are expected to give $R>1$ in the mesoscale range. For $R<1$ linear atmospheric IGWs are ruled out as primary contributors to the kinetic energy since the dynamics is dominated by vortical flows $(\mathrm{Li}$ and Lindborg 2018). It is also evident that for quasigeostrophic dynamics $K^{\phi}$ should be very small compared with $K^{\psi}$; therefore, in regions where $R \ll 1$ balanced regimes are dominating. For $R \simeq 1$ stratified turbulence is a possible candidate.

Figure 5 shows the $R$ variations over the frequency range. The vertical dashed and solid lines, indicating, respectively, the lower and upper limits for the IGW frequency range, are plotted as a reference. The smallest frequency considered is $\omega_{\min }=10^{-1} \mathrm{rad} \mathrm{s}^{-1}$ since for $\omega<\omega_{\min }$ the divergent component has imaginary values that cannot be considered (see also Fig. 4a). For $1 \times$ $10^{-1}<\omega<3 \times 10^{-1} \mathrm{rad} \mathrm{s}^{-1}$ the ratio $R$ lies below the red dashed line, which indicates $R=1$. This part is dominated by the large-scale balanced flow, which is in

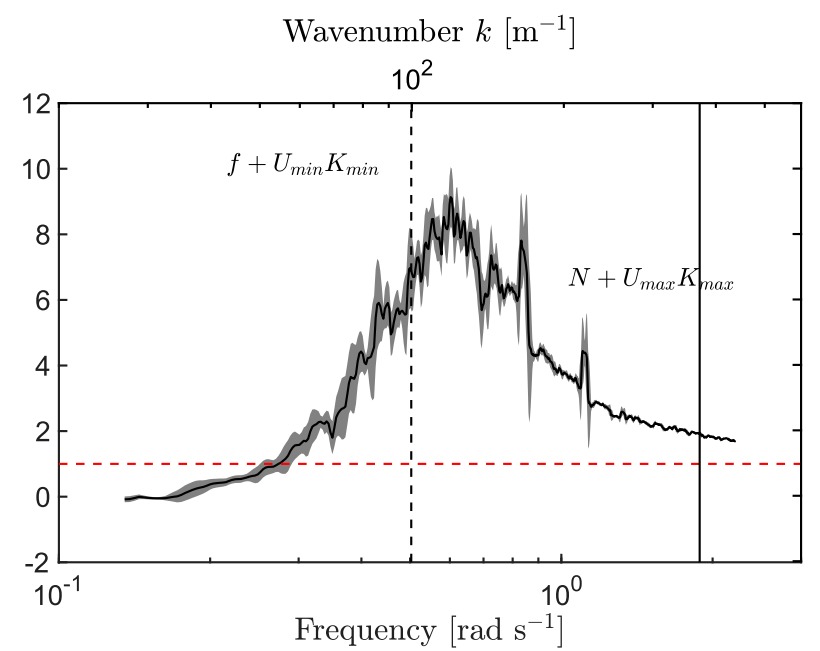

FIG. 5. Ratio between divergent and rotational energy spectra calculated from (6). The black solid line shows $R$ smoothed with a moving average over 10 data points, while the gray shadow shows the variance $2 \sigma$. The red horizontal dashed line indicates $R=1$. The vertical dashed and solid black lines indicates $f+U_{\min } K_{\min }$ and $N+U_{\max } K_{\max }$, respectively, to facilitate the comparison of the frequency ranges with Figs. $4 \mathrm{a}$ and $4 \mathrm{~b}$.

the quasigeostrophic regime. For $\omega>3 \times 10^{-1} \mathrm{rad} \mathrm{s}^{-1}$ the divergent component grows much larger and, therefore, we find $R>1$. Since the slope in Fig. 3 becomes shallower for the same frequency range, the large values for $R$ confirm that gravity waves are, indeed, the main dynamical process underlying the energy at the small scales. For larger frequencies, starting at $\omega \approx 1 \mathrm{rad} \mathrm{s}^{-1}$, the ratio $R$ decreases and tends toward $R=1$. At these smaller scales, we have previously seen that also $K^{\psi}$ enters the shallow part and approaches the $-5 / 3$ slope as well. If this region corresponds to a turbulent regime, the energy should be equally distributed among the geostrophic and the gravity wave modes from which it follows that the geostrophic and ageostrophic spectra should have the same slope (Bartello 2010). A possible interpretation of our finding is that the balanced flow transfers first energy to the imbalanced flow in the form of gravity wave emission. Such waves have smaller scales but are still within the order of magnitude of the Rossby deformation radius and the size of the baroclinic vortices. At even smaller scales there is a forward cascade from the gravity wave scale to even smaller scales that eventually lead to dissipation. In a recent paper, Kafiabad et al. (2019) described the scattering of waves by the background geostrophic turbulence as a potential mechanism behind this forward cascade of energy by waves. They showed this phenomenon leads to the slope of -2 for the wave spectrum, which is consistent with our finding.

Another critical quantity to look at for establishing whether the energy spectra are generated by weakly or 
strongly nonlinear dynamics is the Rossby number. More in detail, the weak nonlinearity condition is verified if the Rossby number is small. Following $\mathrm{Li}$ and Lindborg (2018), we consider the scale-dependent Rossby number defined as

$$
\operatorname{Ro}_{\mathrm{sc}_{i}}=\frac{\sqrt{\langle\delta \mathbf{U} \cdot \delta \mathbf{U}\rangle}}{f d x_{i}}
$$

where the angle brackets \langle\rangle indicate a spatial average, $\delta \mathbf{U}=\mathbf{U}_{x_{1}}-\mathbf{U}_{x_{2}}$ is the velocity difference between two velocities measured at the points $x_{1}$ and $x_{2}, d x=x_{1}-x_{2}$ is the distance between such points, and $f$ is the Coriolis parameter. To calculate $\mathrm{Ro}_{s_{i}}$, we used the radial velocity component and calculated the differences between points along the radial direction. The same operation is started with two adjacent velocities and then repeated increasing stepwise the distance between them until the two points are at the opposite extremes of the domain. This procedure allows us to investigate a range $0.2<d x<30 \mathrm{~cm}$, i.e., from the spatial resolution of our data almost to the entire gap width (some data at the boundaries have been removed because of the significant noise level). The calculation is repeated for the entire time series, and then the mean value is taken.

The resulting $\mathrm{Ro}_{\mathrm{sc}}$ is plotted in Fig. 6, where the red horizontal dashed line marks the value Ro $=1$. The experimental $\mathrm{Ro}_{\mathrm{sc}}$ shows strong similarities with scaledependent Rossby number for stratospheric data (see Fig. 7 in Li and Lindborg 2018). For the smallest scales, Ro $>1$ implying that the dynamics is strongly nonlinear. At scales larger than $3 \mathrm{~cm}$, the Rossby number transitions to values smaller than one. The region by the gray shading marks the gravity wave scales. It follows that at scales for which the Rossby number is smaller than one and $R>1$, linear gravity waves are likely to be responsible for the $k^{-5 / 3}$ spectrum. These findings are in agreement with Li and Lindborg (2018), who concluded that part of the mesoscale spectrum in the lower stratosphere might result from linear gravity waves.

Finally, the marked transition to a nonlinear regime for scales smaller than $3 \mathrm{~cm}$ is in agreement with our hypothesis that there is a transition from nearly linear gravity waves toward a turbulent dynamics at the smaller scales.

\section{Conclusions}

We have presented in this paper an experimental investigation of inertia-gravity wave (IGW) emission from the balanced baroclinic flow in the differentially heated rotating annulus. IGWs are identified in the horizontal divergence field and appear to be emitted

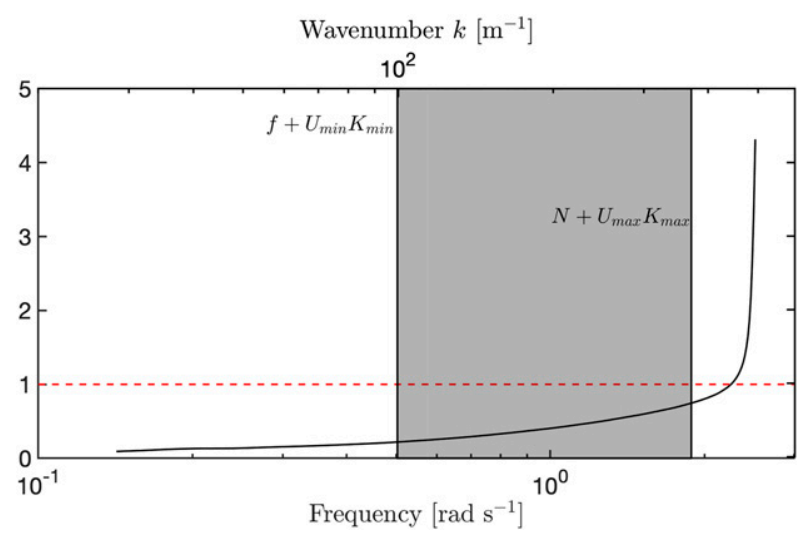

FIG. 6. Scale-dependent Rossby number calculated with (7). The black solid line shows $\mathrm{Ro}_{\mathrm{sc}}$. The horizontal dashed red line marks Ro $=1$. The gray shaded area highlights the gravity wave regime.

from the jet front and subsequently to propagate along with it, mostly advected. The waves in the experiment show several similarities with the atmospheric gravity waves (Suzuki et al. 2013; Dörnbrack et al. 2018). One of the most evident analogies is the wave crest aligned perpendicularly to the jet as a consequence of the wave-capture process they undergo when propagating through regions of the flow with large shear deformation (Plougonven and Snyder 2005).

One open issue is understanding the contribution of IGWs in the midlatitudes to the total energy at the mesoscales. This problem has been debated for more than 30 years, but the analysis of atmospheric data still shows controversial results. Some idealized numerical simulations have been used to investigate stratified turbulence as a possible explanation for the observed mesoscale energy spectrum. However, such models often substantially differ from the atmospheric parameter regimes with buoyancy Reynolds number $\operatorname{Re}_{b} \equiv \operatorname{Fr}_{h}^{2} \operatorname{Re} \sim \mathscr{Q}(1)$, which is much smaller than the typical values for the atmospheric mesoscales $\operatorname{Re}_{b} \sim \mathscr{Q}\left(10^{7}\right)$. For low $\operatorname{Re}_{b}$, the horizontal spectral slope depends upon stratification (Bartello and Tobias 2013). These studies concluded that stratified turbulence without rotation may come short to explain the mesoscale atmospheric spectra (Waite and Bartello 2006). Furthermore, the energy in these models is usually injected at the large scales by some forcing that can be very different from the atmospheric energy forcing (Waite 2014). In our laboratory experiment, the radiative heating represents a more realistic source for the large-scale baroclinic flow, in a regime parameter closer to the real atmosphere, with $\operatorname{Re}_{b} \sim 336$ and rotation playing an important role in the dynamics. Although the experiment still has several simplifications compared to the atmosphere, to the best of the authors' 
knowledge, it is the first laboratory experiment that investigates the energy sources at the mesoscales. The one-dimensional energy spectra obtained from a series of experimental runs in a regular baroclinic wave regime show striking similarities with the atmospheric spectra. The characteristic -3 slope for the large-scale balanced flow turning into a $-5 / 3$ slope for smaller scales is well visible in the kinetic energy spectra. A Helmholtz decomposition is applied to such spectra and reveals that most of the large-scale energy is attributed to the balanced rotational flow, while at the smaller scales, in the subrange with slope $-5 / 3$, the divergent component of the spectrum becomes the most energetic. The ratio between the divergent and the rotational component $R$ has a peak at $R=8$ in the IGW range and then lowers approaching 1 for smaller scales, corresponding to the spectral range where the rotational part approaches a slope of $-5 / 3$.

A possible interpretation of these findings is that the balanced flow undergoes a loss of balance and spontaneously emits inertia-gravity waves to the mesoscale. This process is signaled by a slope change in the energy spectrum, where part of the rotational mode energy is transferred to the divergent modes. Hence, the dynamics at the mesoscale is dominated by inertia-gravity waves. In the $-5 / 3$ region, the energy is cascading forward, being transferred from larger to smaller scales until it dissipates by diffusive processes. Therefore, a further change in the dynamics might occur corresponding to an observed flattening of the vortical component. This scenario with gravity waves dominating the largescale end of the mesoscale, while turbulence becomes prominent at the small-scale end is in agreement with the downscale energy flux observed only at sub-100-km scales by Cho and Lindborg (2001).

Our experimental findings also seem to confirm the results obtained through numerical simulations of an idealized baroclinic wave life cycle by Waite and Snyder (2009). The numerical simulations reveal that spontaneously emitted gravity waves are responsible for the shallower part of the stratospheric spectra (although the same is not observed for the tropospheric spectra). One reason for the alignment of our results with the numerical simulations related to the stratosphere is that, since the lower stratosphere is calmer than the upper troposphere, the stratospheric dynamics might be closer to the regimes we considered in the experiments. Nevertheless, to what extent the processes observed in the experiment apply to the atmosphere is an open issue, since our experiment does not consider, for example, IGWs propagating from the troposphere into the stratosphere, which is a significant source in the atmosphere. One fundamental advantage of doing baroclinic life cycle studies by laboratory experiment is that all the scales up to dissipation are resolved. In the numerical models, instead, the scale smaller than the transition need to be parameterized, and the numerical resolution could significantly influence the mesoscale flow.

In conclusion, we have shown that the differentially heated rotating annulus with a small aspect ratio is not only an analog to the large-scale atmospheric flow, but can also be a useful tool to study multiple-scale processes in atmosphere-like flows and validate numerical simulations and field data.

Acknowledgments. This work was supported by the Spontaneous Imbalance project (HA 2932/8-1 and HA 2932/8-2) that is part of the research group Multiscale Dynamics of Gravity Waves funded by DFG (FOR1898). The authors thank Ludwig Stapelfeld, Robin Stöbel, and Florian Prüfer for technical support. Moreover, we thank the Spontaneous Imbalance group of MS-GWaves, (Ulrich Achatz, Ion Dan Borcia, Steffen Hien, Lena Schoon, Christoph Zülicke) for support and fruitful discussions. The three reviewers are thanked for their insightful comments that help improving and clarifying the manuscript.

\section{REFERENCES}

Bartello, P., 2010: Quasigeostrophic and stratified turbulence in the atmosphere. Symp. on Turbulence in the Atmosphere and Oceans, Cambridge, United Kingdom, International Union of Theoretical and Applied Mechanics, 117-130.

buoyancy Reynolds number. J. Fluid Mech., 725, 1-22, https:// doi.org/10.1017/jfm.2013.170.

Bierdel, L., C. Snyder, S.-H. Park, and W. C. Skamarock, 2016: Accuracy of rotational and divergent kinetic energy spectra diagnosed from flight-track winds. J. Atmos. Sci., 73, 32733286, https://doi.org/10.1175/JAS-D-16-0040.1.

Borchert, S., U. Achatz, and M. D. Fruman, 2014: Gravity wave emission in an atmosphere-like configuration of the differentially heated rotating annulus experiment. J. Fluid Mech., 758 , 287-311, https://doi.org/10.1017/jfm.2014.528.

Bühler, O., and M. E. McIntyre, 2005: Wave capture and wavevortex duality. J. Fluid Mech., 534, 67-95, https://doi.org/ 10.1017/S0022112005004374.

_ J. Callies, and R. Ferrari, 2014: Wave-vortex decomposition of one-dimensional ship-track data. J. Fluid Mech., 756, 10071026, https://doi.org/10.1017/jfm.2014.488.

_- M. Kuang, and E. G. Tabak, 2017: Anisotropic Helmholtz and wave-vortex decomposition of one-dimensional spectra. J. Fluid Mech., 815, 361-387, https://doi.org/10.1017/ jfm.2017.57.

Callies, J., R. Ferrari, and O. Bühler, 2014: Transition from geostrophic turbulence to inertia-gravity waves in the atmospheric energy spectrum. Proc. Natl. Acad. Sci. USA, 111, 17 033-17 038, https://doi.org/10.1073/pnas.1410772111.

Charney, J. G., 1971: Geostrophic turbulence. J. Atmos. Sci., 28, 1087-1095, https://doi.org/10.1175/1520-0469(1971)028<1087: GT>2.0.CO;2. 
Cho, J. Y., and E. Lindborg, 2001: Horizontal velocity structure functions in the upper troposphere and lower stratosphere: 1. Observations. J. Geophys. Res., 106, $10223-10232$, https:// doi.org/10.1029/2000JD900814.

Chouksey, M., C. Eden, and N. Brüggemann, 2018: Internal gravity wave emission in different dynamical regimes. J. Phys. Oceanogr., 48, 1709-1730, https://doi.org/10.1175/JPO-D-17-0158.1.

Dewan, E. M., 1979: Stratospheric wave spectra resembling turbulence. Science, 204, 832-835, https://doi.org/10.1126/ science.204.4395.832.

Dörnbrack, A., M. Pitts, L. Poole, Y. Orsolini, K. Nishii, and H. Nakamura, 2012: The 2009-2010 Arctic stratospheric winter-General evolution, mountain waves and predictability of an operational weather forecast model. Atmos. Chem. Phys., 12, 3659-3675, https://doi.org/10.5194/acp-12-3659-2012.

_ - and Coauthors, 2018: Gravity waves excited during a minor sudden stratospheric warming. Atmos. Chem. Phys., 18, 12 915-12 931, https://doi.org/10.5194/acp-18-12915-2018.

Garrett, C., and W. Munk, 1979: Internal waves in the ocean. Annu. Rev. Fluid Mech., 11, 339-369, https://doi.org/10.1146/ annurev.fl.11.010179.002011.

Hien, S., J. Rolland, S. Borchert, L. Schoon, C. Zülicke, and U. Achatz, 2018: Spontaneous inertia-gravity wave emission in the differentially heated rotating annulus experiment. J. Fluid Mech., 838, 5-41, https://doi.org/10.1017/jfm.2017.883.

Jacoby, T., P. Read, P. D. Williams, and R. Young, 2011: Generation of inertia-gravity waves in the rotating thermal annulus by a localised boundary layer instability. Geophys. Astrophys. Fluid Dyn., 105, 161-181, https://doi.org/10.1080/ 03091929.2011.560151.

Kafiabad, H. A., and P. Bartello, 2016: Balance dynamics in rotating stratified turbulence. J. Fluid Mech., 795, 914-949, https://doi.org/10.1017/jfm.2016.164.

- , and -2017 : Rotating stratified turbulence and the slow manifold. Comput. Fluids, 151, 23-34, https://doi.org/10.1016/ j.compfluid.2016.10.020.

— , and —, 2018: Spontaneous imbalance in the nonhydrostatic Boussinesq equations. J. Fluid Mech., 847, 614643, https://doi.org/10.1017/jfm.2018.338.

_ - M. A. Savva, and J. Vanneste, 2019: Diffusion of inertiagravity waves by geostrophic turbulence. J. Fluid Mech., 869, R7, https://doi.org/10.1017/JFM.2019.300.

Khaykin, S., A. Hauchecorne, N. Mzé, and P. Keckhut, 2015: Seasonal variation of gravity wave activity at midlatitudes from 7 years of COSMIC GPS and Rayleigh lidar temperature observations. Geophys. Res. Lett., 42, 1251-1258, https:// doi.org/10.1002/2014GL062891.

Kumar, A., and M. K. Verma, 2018: Applicability of Taylor's hypothesis in thermally driven turbulence. Roy. Soc. Open Sci., 5, 172152, https://doi.org/10.1098/RSOS.172152.

Li, Q., and E. Lindborg, 2018: Weakly or strongly nonlinear mesoscale dynamics close to the tropopause? J. Atmos. Sci., 75 , 1215-1229, https://doi.org/10.1175/JAS-D-17-0063.1.

Lindborg, E., 2006: The energy cascade in a strongly stratified fluid. J. Fluid Mech., 550, 207-242, https://doi.org/10.1017/ S0022112005008128.

- 2007: Horizontal wavenumber spectra of vertical vorticity and horizontal divergence in the upper troposphere and lower stratosphere. J. Atmos. Sci., 64, 1017-1025, https://doi.org/ 10.1175/JAS3864.1.

, 2015: A Helmholtz decomposition of structure functions and spectra calculated from aircraft data. J. Fluid Mech., 762, R4, https://doi.org/10.1017/JFM.2014.685.
Menchaca, M. Q., and D. R. Durran, 2019: The influence of gravity waves on the slope of the kinetic energy spectrum in simulations of idealized midlatitude cyclones. J. Atmos. Sci., 76, 2103-2122, https://doi.org/10.1175/JAS-D-18-0329.1.

Nastrom, G. D., and K. S. Gage, 1985: A climatology of atmospheric wavenumber spectra of wind and temperature observed by commercial aircraft. J. Atmos. Sci., 42, 950-960, https://doi.org/ 10.1175/1520-0469(1985)042<0950:ACOAWS > 2.0.CO;2.

$\longrightarrow, \ldots$, and W. H. Jasperson, 1984: Kinetic energy spectrum of large- and mesoscale atmospheric processes. Nature, $\mathbf{3 1 0}$, 36-38, https://doi.org/10.1038/310036a0.

O'Sullivan, D., and T. J. Dunkerton, 1995: Generation of inertiagravity waves in a simulated life cycle of baroclinic instability. J. Atmos. Sci., 52, 3695-3716, https://doi.org/10.1175/15200469(1995)052<3695:GOIWIA > 2.0.CO;2.

Plougonven, R., and C. Snyder, 2005: Gravity waves excited by jets: Propagation versus generation. Geophys. Res. Lett., 32, L18802, https://doi.org/10.1029/2005GL023730.

_ , and F. Zhang, 2014: Internal gravity waves from atmospheric jets and fronts. Rev. Geophys., 52, 33-76, https://doi.org/ 10.1002/2012RG000419.

,- H. Teitelbaum, and V. Zeitlin, 2003: Inertia gravity wave generation by the tropospheric midlatitude jet as given by the Fronts and Atlantic Storm-Track Experiment radio soundings. J. Geophys. Res., 108, 4686, https://doi.org/10.1029/ 2003JD003535.

Polichtchouk, I., and R. K. Scott, 2020: Spontaneous inertia-gravity wave emission from a nonlinear critical layer in the stratosphere. Quart. J. Roy. Meteor. Soc., 146, 1516-1528, https:// doi.org/10.1002/qj.3750.

Randriamampianina, A., and E. C. del Arco, 2015: Inertiagravity waves in a liquid-filled, differentially heated, rotating annulus. J. Fluid Mech., 782, 144-177, https://doi.org/ 10.1017/jfm.2015.522.

Read, P. L., E. P. Pérez, I. M. Moroz, and R. M. Young, 2014: General circulation of planetary atmospheres: Insights from rotating annulus and related experiments. Modeling Atmospheric and Oceanic Flows: Insights from Laboratory Experiments and Numerical Simulations, T. von Larcher and P. D. Williams, Eds., John Wiley and Sons, 7-44.

Rodda, C., 2019: Gravity Wave Emission from Jet Systems in the Differentially Heated Rotating Annulus Experiment. Cuvillier Verlag, 200 pp.

- I. Borcia, P. Le Gal, M. Vincze, and U. Harlander, 2018: Baroclinic, Kelvin and inertia-gravity waves in the barostrat instability experiment. Geophys. Astrophys. Fluid Dyn., 112, 175-206, https://doi.org/10.1080/03091929.2018.1461858.

, S. Hien, U. Achatz, and U. Harlander, 2019: A new atmospheric-like differentially heated rotating annulus configuration to study gravity wave emission from jets and fronts. Exp. Fluids, 61, 2, https://doi.org/10.1007/s00348019-2825-z.

Sommeria, J., 2003: Correlation imaging velocimetry at the Coriolis facility. LEGI Rep., 28 pp., http://servforge.legi.grenoble-inp.fr/ projects/soft-uvmat/attachment/wiki/WikiStart/CIV_doc_lim.pdf.

Suzuki, S., K. Shiokawa, Y. Otsuka, S. Kawamura, and Y. Murayama, 2013: Evidence of gravity wave ducting in the mesopause region from airglow network observations. Geophys. Res. Lett., 40, 601605, https://doi.org/10.1029/2012GL054605.

Taylor, G. I., 1938: The spectrum of turbulence. Proc. Roy. Soc. London, 164A, 476-490, https://doi.org/10.1098/rspa.1938.0032.

Tung, K. K., and W. W. Orlando, 2003: The $k^{-3}$ and $k^{-5 / 3}$ energy spectrum of atmospheric turbulence: Quasigeostrophic 
two-level model simulation. J. Atmos. Sci., 60, 824-835, https://doi.org/10.1175/1520-0469(2003)060<0824:TKAKES> 2.0.CO;2.

Vanneste, J., 2013: Balance and spontaneous wave generation in geophysical flows. Annu. Rev. Fluid Mech., 45, 147-172, https://doi.org/10.1146/annurev-fluid-011212-140730.

VanZandt, T., 1982: A universal spectrum of buoyancy waves in the atmosphere. Geophys. Res. Lett., 9, 575-578, https:// doi.org/10.1029/GL009i005p00575.

von Larcher, T., S. Viazzo, U. Harlander, M. Vincze, and A. Randriamampianina, 2018: Instabilities and small-scale waves within the Stewartson layers of a thermally driven rotating annulus. J. Fluid Mech., 841, 380-407, https://doi.org/ 10.1017/jfm.2018.10.

von Storch, J.-S., G. Badin, and M. Oliver, 2019: The interior energy pathway: Inertia-gravity wave emission by oceanic flows. Energy Transfers in Atmosphere and Ocean, C. Eden and A. Iske, Eds., Springer, 53-85.

Waite, M. L., 2014: Direct numerical simulations of laboratoryscale stratified turbulence. Modeling Atmospheric and Oceanic Flows: Insights from Laboratory Experiments and
Numerical Simulations, T. von Larcher and P. Williams, Eds., Wiley, 159-175.

_ , and P. Bartello, 2006: The transition from geostrophic to stratified turbulence. J. Fluid Mech., 568, 89-108, https:// doi.org/10.1017/S0022112006002060.

— of a baroclinic life cycle. J. Atmos. Sci., 66, 883-901, https:// doi.org/10.1175/2008JAS2829.1.

Wang, S., F. Zhang, and C. Snyder, 2009: Generation and propagation of inertia-gravity waves from vortex dipoles and jets. J. Atmos. Sci., 66, 1294-1314, https://doi.org/10.1175/2008JAS2830.1.

Wu, D. L., and F. Zhang, 2004: A study of mesoscale gravity waves over the North Atlantic with satellite observations and a mesoscale model. J. Geophys. Res., 109, D22104, https://doi.org/ 10.1029/2004JD005090.

Yarom, E., and E. Sharon, 2014: Experimental observation of steady inertial wave turbulence in deep rotating flows. Nat. Phys., 10, 510-514, https://doi.org/10.1038/nphys2984.

Žagar, N., D. Jelić, M. Blaauw, and P. Bechtold, 2017: Energy spectra and inertia-gravity waves in global analyses. J. Atmos. Sci., 74, 2447-2466, https://doi.org/10.1175/JAS-D-16-0341.1. 